\title{
A MIP-based biomimetic sensor for the impedimetric detection of histamine in different $\mathrm{pH}$ environments
}

\author{
E. Bongaers ${ }^{* * 1}$, J. Alenus ${ }^{1}$, F. Horemans ${ }^{1}$, A. Weustenraed ${ }^{1}$, L. Lutsen ${ }^{2}$, D. Vanderzande ${ }^{1,2}$, T. J. Cleij ${ }^{1}$, \\ F. J. Troost ${ }^{3}$, R.-J. Brummer ${ }^{3}$, and P. Wagner ${ }^{*, 1,2}$ \\ ${ }^{1}$ Institute for Materials Research, Hasselt University, Wetenschapspark 1, 3590 Diepenbeek, Belgium \\ ${ }^{2}$ IMEC, Division IMOMEC, Wetenschapspark 1, 3590 Diepenbeek, Belgium \\ ${ }^{3}$ Gastroenterology \& Hepatology, Department of Internal Medicine, Nutrition and Toxicology Research Institute Maastricht \\ (NUTRIM), Maastricht University, Universiteitssingel 50, 6229 ER Maastricht, The Netherlands
}

Received 24 September 2009, revised 29 November 2009, accepted 14 December 2009

Published online 19 March 2010

Keywords biosensor, electrochemical sensors, biomaterials

*Corresponding author: e-mail Patrick.Wagner@uhasselt.be, Phone: +32 (0)11 2688 95, Fax: +32 (0) 11268899

** e-mail Evi.Bongaers@uhasselt.be, Phone: +32 (0)11 2688 76, Fax: +32 (0)11 268899

The development of novel biosensors is a rapidly growing field. Substituting the biological receptor layer from the biosensor with a synthetic receptor opens the door for the development of biomimetic sensors that are chemically and physically inert, as opposed to the sensors containing biological recognition elements. Using molecularly imprinted polymers (MIPs) the specificity and affinity of biological receptors can be mimicked. In addition, a MIP-based sensor can measure in harsh environments. Histamine occurs in harsh environments in food and bodily fluids and is chosen as the target molecule for impedimetric detection. When $10 \mathrm{nM}$ histamine is present in $\mathrm{pH}$ neutral environments, the impedance increases $45 \%$ with respect to the impedance of the sensor without histamine. Specificity is tested with respect to histidine. The influence of the $\mathrm{pH}$ on the performance of the sensor is tested. In a $\mathrm{pH}$ range of $\mathrm{pH}$ 5-12 the MIPs are stable, although they exhibit a varying degree of protonation. The same holds true for the target molecule of which the protonation also varies with the $\mathrm{pH}$ of the solution. It is shown that the $\mathrm{pH}$ dependent degree of protonation of both the MIP and the histamine has a large impact on the binding of histamine to the nanocavity in the MIP. Hence, the detection of histamine by a MIP-based sensor is affected by the $\mathrm{pH}$ of the solution.
1 Introduction The development of sensor devices to detect specific molecules is of increasing importance. Innovative biosensors make an early detection of certain disease marking molecules possible. Biosensors make use of biological recognition elements, such as antibodies [1] or enzymes [2]. However, existing sensing systems based on biological recognition elements have restrictions limiting their use [3,4]. First of all, biological recognition elements are not always available for the desired target. Furthermore, they are unstable in organic solvents, high temperature, or changing $\mathrm{pH}$. A synthetic recognition element can be more inert and stable in a wide range of conditions such as temperature or $\mathrm{pH}[5]$.

Molecularly imprinted polymers (MIPs) are synthetic receptors, which exhibit similar specificity and selectivity to the desired target molecules as their natural antibodies or enzymes [5-8]. Substituting the biological recognition elements by a synthetic alternative creates opportunities for the development of biomimetic sensors, which potentially can be applied in harsh environments. MIPs are polymers that contain nanocavities with the exact shape, size, and functional groups (e.g. hydrogen bonds) complementary to their target molecule. MIPs are especially suitable for detection of low molecular weight molecules $[9,10]$. In the presence of the target molecule, binding will occur in the nanocavities of the MIP, thereby changing the dielectric properties of the MIP. These electrical changes can be measured by means of impedance spectroscopy. MIPs have successfully been integrated in impedimetric sensing devices, such as the nicotine sensor [11], and in 
electrochemical [12], piezo-electric [13], and optical sensors [14]. An advantage of an impedimetric sensor is that it allows for electronic data storage and analysis. Impedimetric sensors with biological recognition elements have previously been successfully developed on polymer and diamond transducer platforms $[15,16]$.

MIPs are generally believed to withstand a wide range of $\mathrm{pH}$ conditions. In this article it is investigated how the $\mathrm{pH}$ affects the functionality of the MIP. To this end histamine is chosen as a relevant target molecule. Several food products contain histamine, such as red wine, which has a $\mathrm{pH}$ of 3.4, and rotten fish, which contains elevated levels of histamine. Histamine also plays an important role in irritable bowel syndrome (IBS) [17], allergies [18], asthma [19], rheumatoid arthritis [20], and related diseases. For application of the sensor in bodily fluids a chemical recognition element is necessary, which can sustain the acidic environments of fluids in which histamine occurs, such as stomach fluids, bowel fluids, and saliva. As a result, the development of a histamine sensor containing such a chemical recognition element is of considerable interest. This is especially the case since there is a high demand for fast tests that can detect low concentrations of histamine under a broad range of $\mathrm{pH}$ conditions.

Histamine is derived from the decarboxylation of the amino acid histidine, a reaction which is catalyzed by the enzyme L-histidine decarboxylase. Histamine is a small molecule with a low molecular weight $(111 \mathrm{Da})$ and a chemical formula $\mathrm{C}_{5} \mathrm{H}_{9} \mathrm{~N}_{3}$ [21]. The resembling molecule histidine is used to test specificity of the sensor. Histamine and histidine are both depicted in Fig. 1.

Until present the following techniques have been reported in literature for the analysis of histamine in biological fluids. Fluorometric assays were widely used in tissue [22] and in whole blood and plasma [23, 24, 25]. However, the measurement of a large number of samples is complicated by the laborious extraction steps that are needed. High-speed liquid chromatography can be used to overcome some disadvantages of fluorometric assay, although this requires a substantial amount of material and time [26]. Radioisotopic enzymatic assay is a sensitive method, which is specific in biological fluids [27], however the presence of other compounds inhibits the enzymatic reaction needed for this method of detection, making it difficult to determine histamine levels. As an alternative, gas chromatography-mass spectroscopy [28] fulfills many sensitivity, specificity and accuracy demands. However

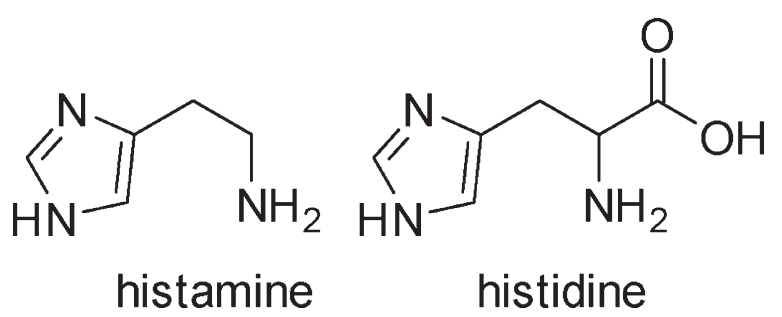

Figure 1 Chemical structure of histamine and histidine. sample manipulation is very complex and instrumentation costs are very high. Other techniques are capillary electrophoresis (CE) [29] and high-performance liquid chromatography (HPLC) [30].

For routine determination of histamine, an immunoassay is suitable for the rapid analysis of large quantities of samples. In an immunoassay histamine is detected by binding specifically with its antibody. Preparation of such an antibody has only become possible in 1984 [31]. The binding of histamine to its antibody can be detected with a radioimmunoassay (RIA). This technique is often used for a wide range of biologically important molecules. In the mean time, successful RIA's for the detection of histamine have been developed ever since [32-35]. The antibody-antigen binding can also be detected colorimetric by means of enzyme-linked immunosorbent assay (ELISA) [36]. Notwithstanding, considering all disadvantages of the above-mentioned techniques it is desirable to develop a sensor that does not require reagents, works as a direct assay, is applicable in "point-of-care" context and gives a fast "real time" result.

2 Materials and methods MIPs are synthesized as follows. A mixture of methacrylic acid (MAA) $(17.8 \mathrm{mmol})$, ethylene glycol dimethacrylate (EGDM) $(36 \mathrm{mmol})$, and azobisisobutyronitrile (AIBN) $(0.66 \mathrm{mmol})$ was dissolved in $7 \mathrm{~mL}$ dimethylsulfoxide (DMSO) together with the template molecule histamine ( $8.99 \mathrm{mmol})$. This mixture was degassed for $5 \mathrm{~min}$ with $\mathrm{N}_{2}$ to remove oxygen. For polymerization the solution was sealed and kept in a thermostatic oil bath at $65^{\circ} \mathrm{C}$ for $12 \mathrm{~h}$. After polymerization the bulk polymer was grounded with a mechanical mortar for $24 \mathrm{~h}$ and sieved through a $25-\mu \mathrm{m}$ sieve. Only particles with a size smaller than $25 \mu \mathrm{m}$ were used. Next, the histamine was removed from the MIP powders by Soxhlet extraction with methanol (48 h), followed by a mixture of acetic acid/acetonitrile (1/1) $(48 \mathrm{~h})$ and finally again methanol (12 h). The extracted MIP powders were dried in vacuum for $12 \mathrm{~h}$. A schematic representation has been made in Fig. 2. A non-imprinted polymer (NIP) was synthesized in the same manner, but without the presence of the target molecule.

The impedimetric sensor consists of four coplanar aluminum electrodes of $70 \mathrm{~nm}$ thickness and an interspace of $0.6 \mathrm{~mm}$ evaporated onto glass substrates. They are coated with a thin polymer film $(200 \mathrm{~nm})$ of MDMO-PPV (Poly[2methoxy-5-(3,7-dimethyloctyloxy)-1-4-phenylene vinylene). This PPV-derivative is synthesized via the sulphinyl precursor route $[37,38]$ and this semiconducting polymer not only serves as a transducer layer but also as an immobilization layer for the MIP particles. Besides MIP particles, NIP particles also can be immobilized on the transducer layer as reference for measuring non-specific absorption to the polymer film. Each electrode can be individually functionalized by applying the desired microparticles to the polymer layer using the stamping method. With a polydimethylsiloxane (PDMS) stamp, with the same shape and size of the coplanar electrode couple, 

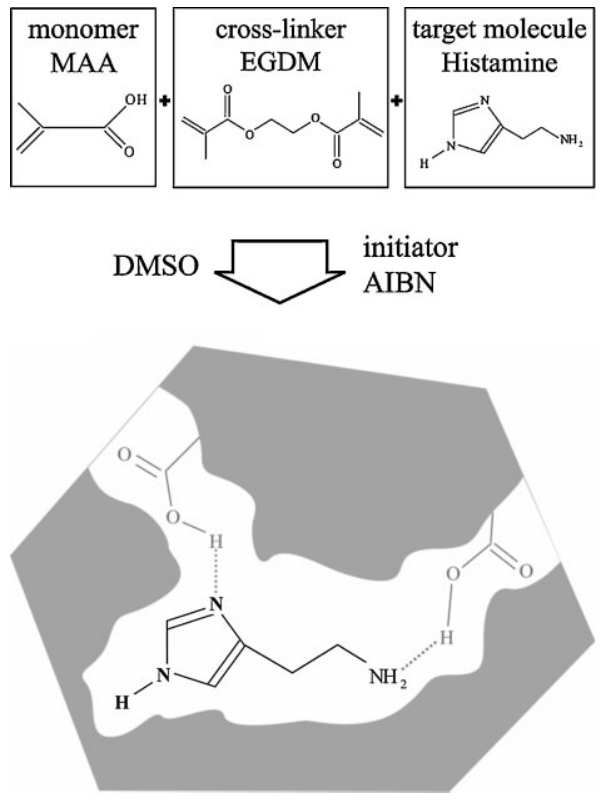

Figure 2 Schematic representation of MIP synthesis with histamine as target molecule.

microparticles are placed onto the polymer surface and placed on a hot plate at $120^{\circ} \mathrm{C}$ for $10 \mathrm{~min}$. This temperature is above the glass transition temperature of MDMO-PPV and will cause the MIP and NIP particles to partially embed into the MDMO-PPV layer. Substrates are then cooled down to room temperature, rinsed with isopropanol to remove excessive and non-embedded MIP and NIP microparticles and dried under nitrogen gas.

The MIP-sensitized electrodes are integrated in an electrochemical cell consisting of a printed circuit board (PCB) and a $500 \mu l$ Teflon reservoir on top. Contacts are wirebonded and the reservoir contains a hole on top for easy addition of analyte concentrations. During the measurement the hole is covered. A PDMS ring is used between sample and hood to prevent leaking. This electrochemical measuring device, shown in Fig. 3, contains four channels, allowing for simultaneous reference measurements with NIPs. The addition setup is placed in a temperature-controlled environment $\left(37^{\circ} \mathrm{C}\right)$ shielded from light.

Impedance spectroscopy was performed using an Iviumstat electrochemical analyzer from Ivium Technologies B.V. For each sensing spot, spectra were measured sequentially at a low-frequency range from $1 \mathrm{~Hz}$ to $1 \mathrm{kHz}$ with zero bias voltage and an oscillating voltage of $50 \mathrm{mV}$. Time-resolved analysis of the impedance spectra is performed in the low frequency range. At low frequencies the sensor is more sensitive for surface interactions between the MIP binding sites, the target molecules and the electrolyte. It is found that upon binding, electrical changes occur at the interface [11]. A double layer is formed at the electrode surface, which is reflected in the capacitive changes at the lower frequency range. Even more, this double layer affects the charge transfer from the electrolyte to the electrode. In this most useful frequency range a frequency of $213 \mathrm{~Hz}$ was chosen for its optimal signal to noise ratio and stability.

The target molecule histamine was obtained from Aldrich. To test specificity of the MIPs the analog molecule histidine was used, obtained from Fluka. A dilution range of histamine and histidine is made in a phosphate buffered saline (PBS) solution. For the dose response curve the concentration of histamine was increased in a 0 to $12 \mathrm{nM}$ range. This is achieved by preparing a histamine concentration range of 9 to $31 \mathrm{nM}$ in 12 equal steps. By sequentially adding constant volumes of $25 \mu \mathrm{l}$ of these concentrations to the $200 \mu \mathrm{l}$ PBS inside the reservoir $1 \mathrm{nM}$ increments of concentrations are obtained from 0 to $12 \mathrm{nM}$. The histidine concentrations were increased by repeated addition of $50 \mathrm{ml}$ of a $29 \mathrm{nM}$ solution, resulting in unequal steps of 5.8, 9.7, and $12.4 \mathrm{nM}$. The dose response measurements occur at a $\mathrm{pH}$ of 7 (neutral environment).

MIPs are known to sustain acid and basic environments very well and are therefore very suitable for application in biosensors for measuring in vivo. Now the detection of histamine by the MIP-based sensor is tested in various $\mathrm{pH}$ environments. PBS with $\mathrm{pH} 5,7,9$, and 12 are prepared by adding small quantities of $\mathrm{HCl}$ or $\mathrm{NaOH}$ to the neutral buffer solution. A histamine concentration series is created with these PBS solutions in order to obtain three equal steps of 3, 6 and $9 \mathrm{nM}$. The three subsequent addition steps of 3,6 and $9 \mathrm{nM}$ are labeled from I to III. The fewer but larger steps result in shorter measuring while a similar concentration range is maintained.

3 Results and discussion To translate the chemical recognition occurring at the MIP and NIP covered surfaces directly into an electrical signal, impedance spectroscopy is performed. Therefore the previously described addition

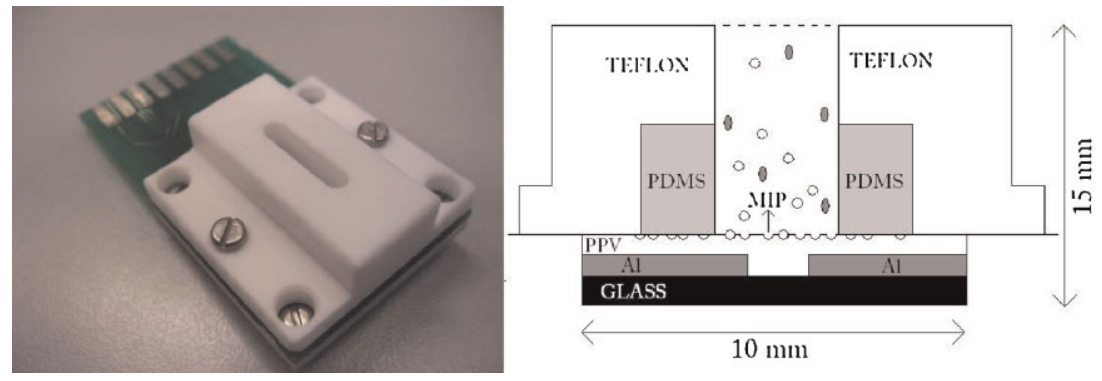

Figure 3 (online color at: www.pss-a.com) Addition setup containing a Teflon hood (left) and a schematic representation of the crosssection (right). 


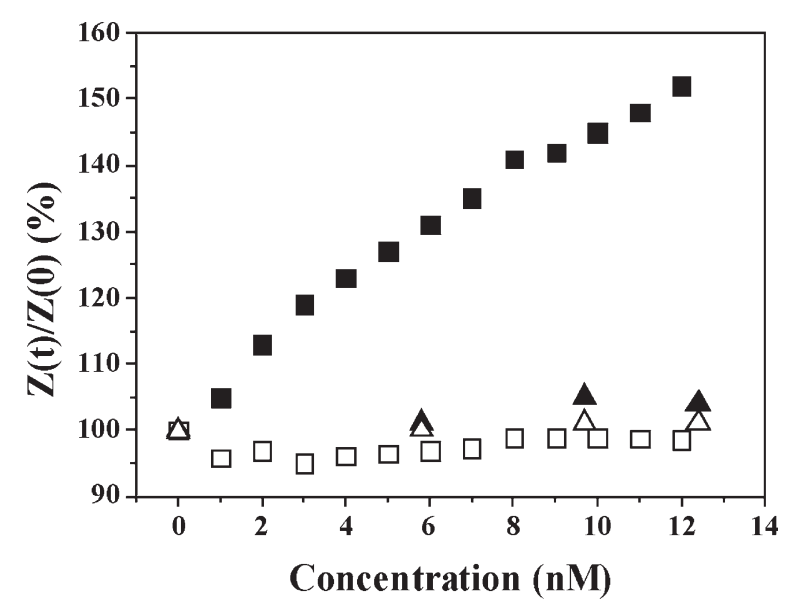

Figure 4 Dose response curves of MIP and NIP channels in response to addition of histamine and histidine obtained with impedance measurements. (MIP exposed to histamine: solid squares; MIP exposed to histidine: solid triangles; NIP exposed to histamine: open squares; NIP exposed to histidine: open triangles).

setup is filled with PBS to simulate a biological environment. After a stabilization period of $15 \mathrm{~min}$, increasing concentrations of histamine are added stepwise. Impedance spectra are measured subsequently and out of four measured channels the two most stable ones are chosen for analysis. At a selected frequency of $213 \mathrm{~Hz}$ a clear dose-response curve is obtained in the $0-12 \mathrm{nM}$ concentration range (Fig. 4). It should be noted that all impedance data are normalized relative to their initial impedance value, prior to addition of the target molecule. The resulting value is referred to as the relative impedance signal $\mathrm{Z}(t) / \mathrm{Z}(0)$. A response value is obtained $20 \mathrm{~min}$ after addition of the target molecule by averaging five data points of which two before and two after the data point at $20 \mathrm{~min}$. The error bars obtained in this manner are an indication for the stability of the measured impedance signal. The actual error bars in Fig. 4 are smaller than the symbols utilized, indicating a low noise level. At $213 \mathrm{~Hz}$ the MIP-sensor shows a 45\% response to a concentration of $10 \mathrm{nM}$ histamine. At higher concentrations the sensor will reach its saturation level. The NIP channel, which measures non-specific binding of histamine molecules to the sensor surface, shows no sensitivity toward histamine.

In addition, the specificity is tested with the analog molecule histidine. At $213 \mathrm{~Hz}$ both the MIP and NIP channel show no response to histidine concentrations in the same nanomolar range. For a better graphical representation of the specific binding events occurring at the sensor surface, the relative impedance signal of the MIP channel is subtracted from the relative impedance signal NIP channel, which is associated with non-specific binding (Fig. 5). It can be seen that the histamine MIP-sensor exhibits a $40 \%$ response to $10 \mathrm{nM}$ histamine. In addition, the sensor response can be represented by a simple allometric fit, as described by Eq. (1), where $x$ is the concentration of target molecule and $y$ the impedance signal representing the amount of target molecule

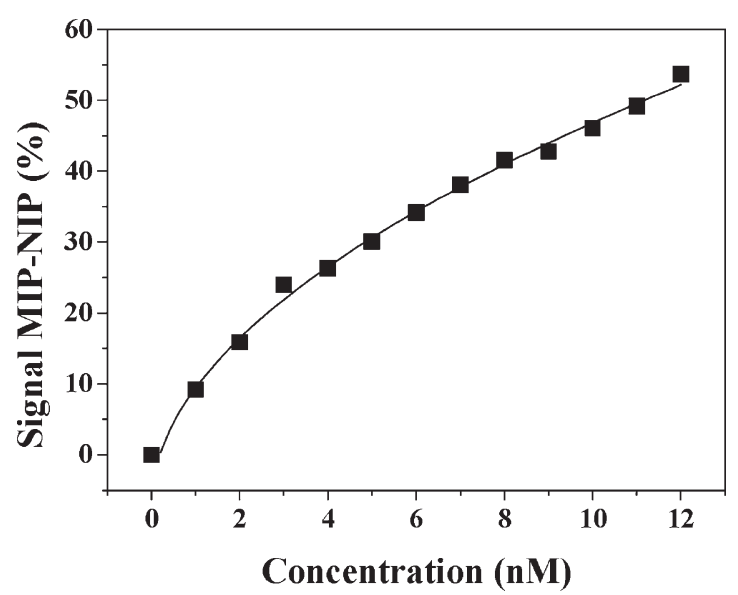

Figure 5 Difference between the relative impedance signals of the MIP channel and the NIP channel. The resulting fit is a good measure for the specific binding occurring at the sensor surface.

that has specifically bonded to the MIP.

$$
y=a x^{b} .
$$

An allometric fit reflects a typical binding isotherm. Each binding site has a different affinity and such heterogeneous distribution of the binding sites results in a binding isotherm which is best analyzed with the Freundlich model, displayed in Eq. (2) [11 and references there in]. In this model $q$ is the amount of bound target molecules per gram of adsorbent (MIP) and $C_{f}$ the concentration of free target molecules in the supernatant fluid. $K_{f}$ is the Freundlich constant and $v$ the Freundlich heterogeneity parameter. The fitted values are displayed in Table 1.

$$
q=K_{f} C_{f}^{v} .
$$

For the envisioned application in body fluids the sensor is tested under various $\mathrm{pH}$ conditions. The results are shown in Fig. 6. In an acidic solution of $\mathrm{pH} 5$ the sensor exhibits no response to the addition of histamine in both the MIP channel and the NIP channel (Fig. 6a). In neutral environments ( $\mathrm{pH} 7$ ), the MIP channel exhibits a response to the subsequent additions of histamine, while the NIP channel does not exhibit non-specific absorption (Fig. 6b). When a concentration of $9 \mathrm{nM}$ is present in the setup, the signal has increased $12 \%$ after $20 \mathrm{~min}$. This is similar to the observations in the dose-response experiments described previously. However, in a basic solution of $\mathrm{pH} 9$ the sensor exhibits an increased response (Fig. 6c). When the concentration is increased to

Table 1 Parameters of the allometric fit of the differential dose response curve.

\begin{tabular}{ll}
\hline parameter & fitted value \\
\hline$a$ & $10.8 \pm 0.5$ \\
$b$ & $0.63 \pm 0.02$ \\
$R^{2}$ & 0.99 \\
\hline
\end{tabular}


a)

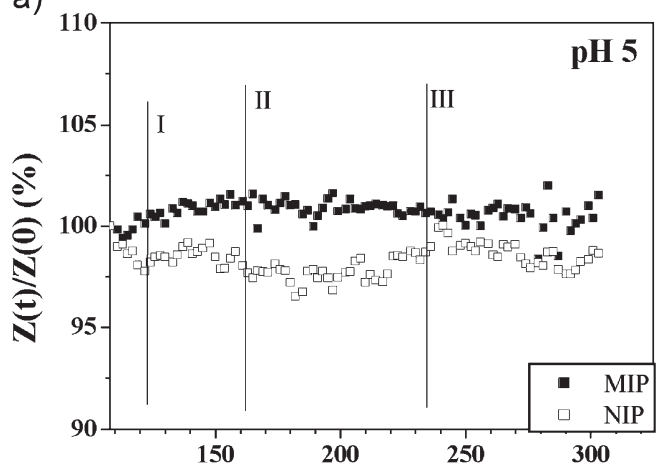

b)

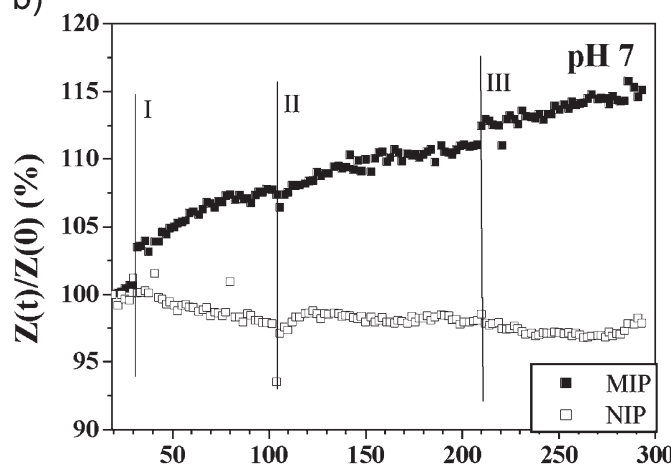

c)
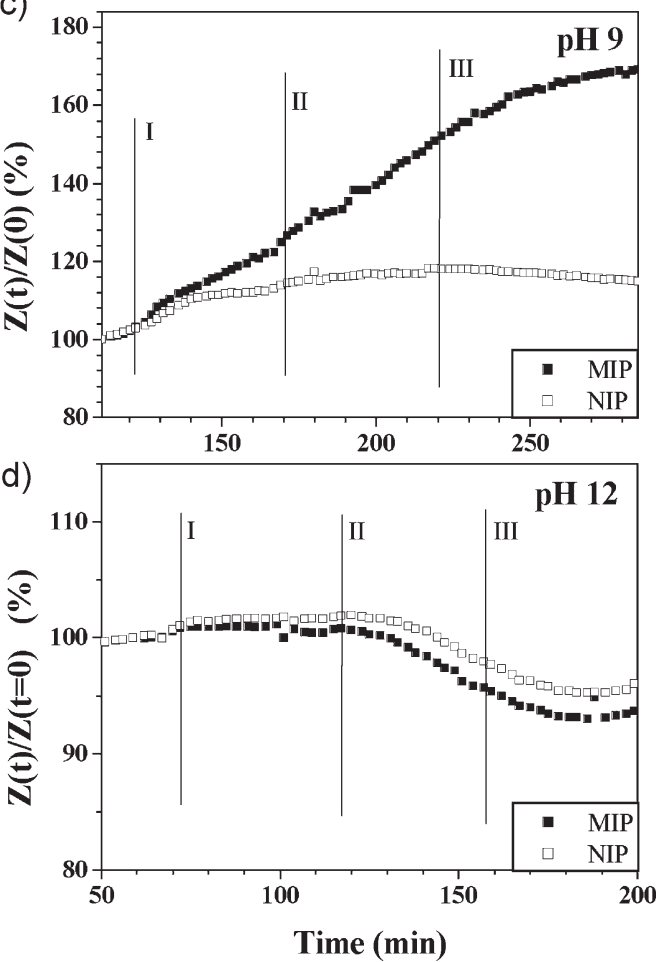

Figure 6 Relative impedance signal of MIP and NIP channels in response to addition of histamine under various $\mathrm{pH}$ conditions: (a) $\mathrm{pH}$ 5, (b) $\mathrm{pH}$ 7, (c) $\mathrm{pH}$ 9, and (d) $\mathrm{pH} 12$. Additions of $3 \mathrm{nM}, 6 \mathrm{Nm}$, and $9 \mathrm{nM}$ are labeled I, II, and III, respectively. (MIP exposed to histamine: solid squares; NIP exposed to histamine: open squares).
$9 \mathrm{nM}$ in the third addition step, after 20 min the sensor signal increases $60 \%$. Also the NIP channel shows very little nonspecific absorption. Finally, the same measurement has been performed in a more basic environment of $\mathrm{pH} 12$ (Fig. 6d). Both signals remain constant after the first addition. After the second and third addition step both the MIP and the NIP channel show a slight signal drift, caused by minor disturbances in the addition setup when analyte is added. Most importantly, in this experiment no specific response of the MIP channel is noticeable as a result of the three subsequent addition steps.

This $\mathrm{pH}$ dependency of the sensor response can be explained by the behavior of the target molecule and the MIP in varying $\mathrm{pH}$ conditions. The appearance of certain molecules depends on the $\mathrm{pH}$ of the solution in which it is dissolved [39]. In an acidic environment protons from the solution might bind to molecules, resulting in protonated molecules. This ionization occurs at a specific $\mathrm{pH}$ value. Histamine has pKa values of 6.9 and 10.4 [40], which are improved values as compared to the ones previously reported in the literature [41]. A third pKa value of about 15-20 has been reported, but this value is less relevant since it does not fall within the $\mathrm{pH}$ range of aqueous solutions [40]. The behavior of histamine in various $\mathrm{pH}$ solutions is modeled with hyperquad simulation and speciation (HySS) [42], which can be used for the investigation of equilibria involving soluble and partially soluble species. The result is depicted in Fig. 7.

In solution, histamine becomes protonated at specific sites. They are depicted in Fig. 8. In strongly basic environments above $\mathrm{pH} \mathrm{10.4,} \mathrm{histamine} \mathrm{will} \mathrm{be} \mathrm{predomi-}$ nantly present in its neutral form (Fig. 1). Upon lowering the $\mathrm{pH}$, the aliphatic amino group will be able to increasingly bind a proton, creating single-protonated histamine or histamine $^{+}$. At $\mathrm{pH} 10.4$, when the $\mathrm{pH}$ is exactly the $\mathrm{pKa}$ value, the neutral histamine and the single-protonated histamine are present in equal concentrations. In neutral environments ( $\mathrm{pH} \mathrm{7)}$ histamine is still mostly present in its single protonated form. However, decreasing the $\mathrm{pH}$ of the buffer solution will further increase the protonation of histamine. At lower $\mathrm{pH}$, the imidazole ring can bind a proton and hence a double protonated form of histamine, histamine $^{++}$will be formed. At pH 6.9 the single protonated and double protonated histamine are present in equal concentrations. Below pH 6.9 double protonated histamine is predominant in the buffer solution. Not only the histamine will be affected by changing the $\mathrm{pH}$, but also the MIP will exhibit protonation and deprotonation reactions. As a model for the MIP, PMAA, poly methacrylic acid, can be used, which can be obtained from the same monomer, i.e. MAA, which is also used to make the MIP. PMMA has a pKa value between 6 and 7 [43]. Its protonation behavior can also be modeled using HySS as depicted in Fig. 9.

For a successful selective binding event of histamine to the MIP, at least one, but preferably more, hydrogen bond has to be formed between histamine and the MIP. This binding will ultimately result in detection in the sensor. However, the 


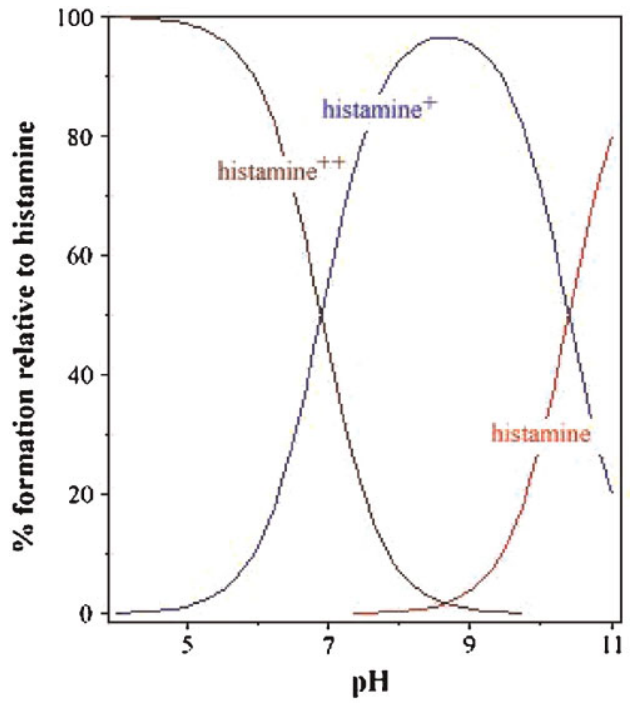

Figure 7 (online color at: www.pss-a.com) Equilibrium of an aqueous solution of histamine at varying $\mathrm{pH}$ values, modeled with HySS [42].

formation of hydrogen bonds is strongly $\mathrm{pH}$ dependent, which explains the results from Fig. 6 as follows. In buffer solutions of $\mathrm{pH} 5$, histamine is mostly double protonated (histamine $^{++}$) and there are almost no histamine ${ }^{+}$molecules left. Since in such acidic solutions also the MIP is almost fully protonated, only very weak hydrogen bonding is possible between the target molecule and the MIP. As a result, the molecule now is unlikely to bind to the nanocavity of the MIP and hence the sensor shows no response to addition of this molecule. At $\mathrm{pH} 7$ the MIP is partially deprotonated. At the same time, about $44 \%$ of the histamine remains double protonated, while the remainder has become single protonated. The histamine ${ }^{+}$and the histamine ${ }^{++}$ molecules can form hydrogen bonds with the partially deprotonated MIP. As a result, the sensor shows a good response at $\mathrm{pH} 7$.

\section{histamine}<smiles>NCCC1CCNC1</smiles><smiles></smiles><smiles>[H][Y]N</smiles>

Figure 8 (online color at: www.pss-a.com) Protonation sites of histamine [40].

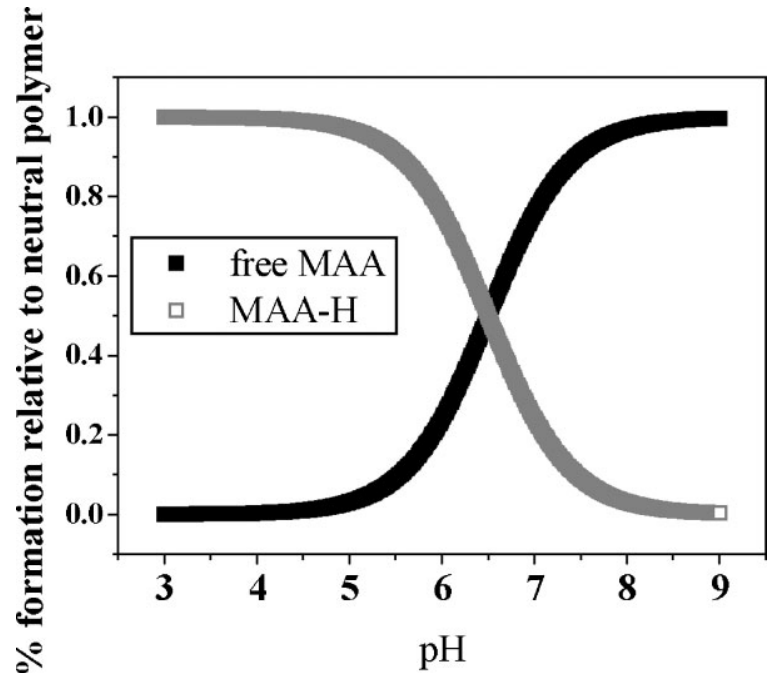

Figure 9 Equilibrium of polymer in solutions, modeled with HySS [41].

At even higher $\mathrm{pH}(\mathrm{pH} 9)$ histamine $^{+}$is predominant, while the MIP is now almost fully deprotonated. This allows the formation of a hydrogen bond and thus the sensor shows a strong response. It is assumed that this condition is close to the optimal balance between protonation of histamine and deprotonation of the polymer.

At $\mathrm{pH} 12$ histamine $^{+}$has mostly been converted into neutral histamine, which is now the predominant molecule. Since, both histamine and the MIP have deprotonized, no large amount of hydrogen bonds can be formed. The MIP channel shows no response to the analyte addition at a $\mathrm{pH}$ of 12 and no specific match with the nanocavity is established.

The long-term stability of this sensor needs to be further tested.

4 Conclusion A fast and specific test has been developed for detecting low concentrations of histamine in the nanomolar range. For example, typical physiological conditions in mast cells are around $200 \mathrm{nM}$. The impedimetric MIP-based sensor has a limit of detection of $2 \mathrm{nM}$. The sensitivity is $45 \%$ to $10 \mathrm{nM}$ histamine. A dose response curve is measured in the $0-12 \mathrm{nM}$ range. Specificity was confirmed by tests with histidine, showing no significant sensor response.

The detection of the MIP-based sensor is affected by the $\mathrm{pH}$ of the environment. Although the MIP can withstand a wide range of $\mathrm{pH}$ values, the $\mathrm{pH}$ of the electrolyte affects protonation or deprotonation of target molecule and MIP. This has a substantial impact on the formation of hydrogen bonds, which are needed for binding of the target molecule to the MIP.

Acknowledgements The authors kindly acknowledge financial support from the transnational University Limburg, from IMEC via a $\mathrm{PhD}$ fellowship and from Hasselt University through the BOF-project "Development and characterization of 
fluorescent MIPs for nanotechnology and applications in advanced electronic devices, chemo- and biosensors". The authors would like to thank to Peter Gans from Protonic software for his help with HySS. Technical assistance by J. Sogen and J. Baccus (Hasselt University) is gratefully acknowledged.

\section{References}

[1] A. P. F. Turner, Nat. Biotechnol. 15, 421 (1997).

[2] I. Lapenaite, B. Kurtinaitiene, L. Marcinkeviciene, L. Bachmatova, V. Laurinavicius, and A. Ramanavicius, Chem. Pap. 55, 345-349 (2001).

[3] V. B. Kandimalla and H. Ju, Anal. Bioanal. Chem. 380, 587605 (2004).

[4] K. Yano and I. Karube, Trends Anal. Chem. 18, 199-204 (1999).

[5] P. K. Owens and L. Karlsson, Trends Anal. Chem. 18, 146154 (1999).

[6] S. A. Piletsky, S. Alcock, and A. P. F. Turner, Trends Biotechnol. 19, 9-12 (2001).

[7] K. Haupt and K. Mosbach, Trends Biotechnol. 16, 468-475 (1998).

[8] K. Haupt and K. Mosbach, Chem. Rev. 100, 2495-2504 (2000).

[9] S. A. Piletsky, N. W. Turner, and P. Laitenberger, Med. Eng. Phys. 28, 971-977 (2006).

[10] F. L. Dickert, P. Lieberzeit, S. G. Miarecka, K. J. Mann, O. Hayden, and C. Palfinger, Biosens. Bioelectron. 20, 10401044 (2004).

[11] R. Thoelen, R. Vansweevelt, J. Duchateau, F. Horemans, J. D'Haen, L. Lutsen, D. Vanderzande, M. Ameloot, M. vandeVen, T. J. Clei, and P. Wagner, Biosens. Bioelectron. 23, 913-918 (2008).

[12] M. C. Blanco-López, M. J. Lobo-Castañón, A. J. MirandaOrdieres, and P. Tuñón-Blanco, Trends Anal. Chem. 23, 3647 (2004).

[13] M. Avila, M. Zougagh, A. Rios, and A. Escarpa, Trends Anal. Chem. 27, 54-65 (2008).

[14] S. A. Piletsky, E. V. Piletskaya, A. V. El'skaya, R. Levi, K. Yano, and I. Karube, Anal. Lett. 30, 445-455 (1997).

[15] P. Cooreman, R. Thoelen, J. Manca, M. vandeven, V. Vermeeren, L. Michiels, M. Ameloot, and P. Wagner, Biosens. Bioelectron. 20, 2151-2156 (2005).

[16] V. Vermeeren, N. Bijnens, S. Wenmackers, M. Daenen, K. Haenen, O. A. Williams, M. Ameloot, M. vandeVen, P. Wagner, and L. Michiels, Langmuir 23, 13193-13202 (2007).

[17] G. Barbara, V. Stanghellini, R. De Giorgio, C. Cremon, G. S. Cottrell, D. Santini, G. Pasquinelli, A. M. Morselli-Labate, E. F. Grady, N. W. Bunnett, S. M. Collins, and R. Corinaldesi, Gastroenterology 126, 693-702 (2004).

[18] R. Y. Lin, L. B. Schwartz, A. Curry, G. R. Pesola, R. J. Knight, H. S. Lee, L. Bakalchuk, C. Tenenbaum, and R. E. Westfal, J. Allergy Clin. Immunol. 106, 65-71 (2000).

[19] J. Chhabra, Y.-Z. Li, H. Alkhouri, A. E. Blake, Q. Ge, C. L. Armour, and J. M. Hughes, Eur. Respir. J. 29, 861-870 (2007).
[20] M. Adlesic, M. Verdrengh, M. Bokarewa, L. Dahlberg, S. J. Foster, and A. Tarkowski, Scand. J. Immunol. 65, 530-537 (2007).

[21] M. A. Beaven, Monogr. Allergy 13, 1-113 (1978).

[22] P. A. Shore, A. Burkhalter, and V. H. Cohn, Jr, J. Pharmacol. Exp. Ther. 127, 182-186 (1959).

[23] W. Lorenz, H. J. Reimann, H. Barth, J. Kusche, R. Meyer, A. Doenicke, and M. Hutzel, Hoppe-Seyler's Z. Physiol. Chem. 353, 911-920 (1972).

[24] P. A. Shore, A. Burkhalter, and V. H. Cohn, Jr., J. Pharmacol. Exp. Ther. 127, 182-186 (1959).

[25] R. P. Siraganian, J. Immunol. Methods 7, 283-290 (1975).

[26] Y. Tsuruta, K. Kohashi, and Y. Ohkura, J. Chromatogr. 146, 490-493 (1978).

[27] M. A. Beaven, S. Jacobsen, and Z. Horakova, Clin. Chim. Acta 37, 91-103 (1972).

[28] J. J. Keyzer, B. G. Wolthers, F. A. J. Muskiet, H. Breukelman, H. F. Kauffman, and K. de Vries, Anal. Biochem. 139, 474 481 (1984).

[29] F. Nishiwaki, K. Kuroda, Y. Inoue, and G. Endo, Biomed. Chromatogr. 14, 184-187 (2000).

[30] V. Van Vietinghoff, G. Gäbel, and J. R. Aschenbach, J. Chromatogr. B Analyt. Technol. Biomed. Life Sci. 844, 335-339 (2006).

[31] H. Mita, H. Yasueda, T. Shida, and S. Baba, Agents Actions 14, 5-6 (1984).

[32] H. L. Waldum, A. K. Sandvik, E. Brenna, and B. Schulze Sognen, Scand. J. Gastroenterol. Suppl. 180, 32-39 (1991).

[33] M. Yamaguchi, K. Sayama, K. Yano, C. S. Lantz, N. NobenTrauth, C. Ra, J. J. Costa, and S. J. Galli, J. Immunol. 162, 5455-5465 (1999).

[34] A. W. Van Toorenenbergen and A. M. Vermeulen, Inflamm. Res. 30, 278-280 (1990).

[35] B. Rotz, A. Savaser, I. Werthmann, S. Lau, and U. Wahn, Allergy 46, 529-533 (1991).

[36] A. Ujike, Y. Ishikawa, M. Ono, T. Yuasa, T. Yoshino, M. Fukumoto, J. V. Ravetch, and T. Takai, J. Exp. Med. 189, 1573-1578 (1999).

[37] A. J. J. M. van Breemen, D. J. M. Vanderzande, P. J. Adriaensens, and J. M. J. V. Gelan, J. Org. Chem. 64, 3106-3112 (1999).

[38] A. J. J. M. van Breemen, A. C. J. Issaris, M. M. de Kok, M. J. A. N. Van Der Borght, P. J. Adriaensens, J. M. J. V. Gelan, and D. J. M. Vanderzande, Macromolecules 32, 5728-5735 (1999).

[39] T. B. Paiva, M. Tominaga, and A. C. M. Paiva, J. Med. Chem. 13, 689-692 (1970).

[40] H. A. De Abreu, W. B. De Almeida, and H. A. Duarte, Chem. Phys. Lett. 383, 47-52 (2004).

[41] F. Holmes and F. Jones, Chem. Soc. 2818-2836 (1962).

[42] L. Alderighi, P. Gans, A. Ienco, D. Peters, A. Sabatini, and A. Vacca, Coord. Chem. Rev. 184, 311-318 (1999).

[43] V. A. Izumrudov, E. Kharlampieva, and S. A. Sukhishvili, Biomacromolecules 6, 1782-1788 (2005). 